hep-ph/yymmddd

\title{
Various definitions of Minimal Flavour Violation for Leptons
}

\author{
Sacha Davidson ${ }^{1, *}$ and Federica Palorini ${ }^{1, \dagger}$ \\ ${ }^{1}$ IPNL, Université CB Lyon-1, 4 rue Enrico Fermi, 69622 Villeurbanne,France
}

\begin{abstract}
Neutrino masses imply the violation of lepton flavour and new physics beyond the Standard Model. However, flavour change has only been observed in oscillations. In analogy with the quark sector, we could deduce the existence of a principle of Minimal Flavour Violation also for Leptons. Such an extension is not straightforward, since the mechanisms generating neutrino masses are unknown and many scenarios can be envisaged. Thus, we explore some possible definitions of MFVL and propose a notion that can include many models. We build an R-parity violating neutrino mass model in agreement with our preferred definition of MFVL, and show that flavour violating processes are not neccessarily controlled by the MNS mixing matrix.
\end{abstract}

\section{Introduction}

Minimal flavour violation [1, 2] in the quark sector, is a useful framework in which to construct TeV-scale models of New Physics. It is predictive, and includes many or most models that are consistent with quark flavour data. Recently, a definition of Minimal Flavour Violation (MFV) has been introduced for leptons [3]. The proposed formulation is predictive - it implies that lepton flavour violation is determined by the light neutrino mass matrix - but includes few of the many neutrino mass models [4, 5, 6, 7, 8, 9] that are consistent with current observations.

The flavour-changing mixing angles of the leptonic sector (MNS matrix), are not measured with the overconstrained precision of the CKM matrix. So MFV is not strongly suggested for leptons, as it is for quarks. However, if one assumes that there is new physics at the TeV-scale, that satisfies MFV or a similar principle in the quark sector, then it is reasonable to expect a similar principle to apply for leptons. So it is interesting to explore different possible definitions of minimal flavour violation for leptons (MFVL), and in particular to study whether it implies that lepton flavour violation is controlled by the MNS matrix and the light neutrino masses.

In this paper, we take the principle of MFV to limit the number of flavour structures allowed to the renormalisable couplings of the theory. This flexible definition can be applied to many models, but is less predictive than [3. We explicitly construct an R-parity violating neutrino mass model that is "minimally flavour violating", in agreement with observation, and where the lepton flavour violation is not controlled by the light neutrino mass matrix.

In section 2, we review minimal flavour violation for the quarks, and classify neutrino mass generation mechanisms. In section 3 we discuss the purpose of Minimal Flavour Violation for leptons, and various possible implementations which we apply to some neutrino mass models. In section 4 we build an R-parity violating neutrino mass model, using the $\lambda L L E^{c}$ coupling, that satisfies our preferred definition of MFVL. In the Appendix is sketched a model satisfying a more restrictive definition of MFVL.

\section{Review}

Beyond-the-Standard-Model physics, in the form of new particles or new interactions, must exist at some scale, to explain observations such as dark matter, neutrino masses, the baryon asymmetry and the temperature fluctuations in the Cosmic Microwave Background. New physics at the TeV-scale (such as, for instance, supersymmetry) is particularily desirable because it could be discovered at the LHC, and would be theoretically welcome to address the hierarchy problem. However, if there are new flavoured TeV-scale particles, as one would like, it is puzzling that their footprints have not been seen in rare flavoured and CP violating processes. So Minimal Flavour Violation is introduced as a constraint on the interactions of such new particles, to suppress their contributions to flavoured observables.

\footnotetext{
*E-mail address: s.davidson@ipnl.in2p3.fr

${ }^{\dagger}$ E-mail address: f.palorini@ipnl.in2p3.fr
} 
We follow the approach to Minimal Flavour Violation of [1], which starts from the flavour transformation properties of various terms in the SM Lagrangian. We define the SM to have massless neutrinos. In three generations, the fermionic kinetic terms

$$
\bar{q}_{L} \not D q_{L}+\bar{u}_{R} \not D u_{R}+\bar{d}_{R} \not D d_{R}+\bar{\ell} \not D \ell+\bar{e}_{R} \not D e_{R}
$$

have a global $U_{q}(3) \times U_{u}(3) \times U_{d}(3) \times U_{\ell}(3) \times U_{e}(3)$ flavour symmetry. For instance, $q_{L}$ is a three component vector in quark doublet flavour space, whose kinetic term is invariant under $q_{L} \rightarrow V_{q} q_{L}$, where $V_{q} \in U_{q}(3)$. This large symmetry group is broken to $U_{B}(1) \times U_{L_{e}}(1) \times U_{L_{\mu}}(1) \times U_{L_{\tau}}(1)$ by the Yukawa couplings

$$
\bar{q}_{L} \mathbf{Y}_{\mathbf{u}} H_{u} u_{R}+\bar{q}_{L} \mathbf{Y}_{\mathbf{d}} H_{u}^{c} d_{R}+\bar{\ell} \mathbf{Y}_{\mathbf{e}} H_{u}^{c} e_{R}+\text { h.c. }
$$

where $H_{u}$ is the SM Higgs, and the index order on Yukawa matrices is left-right. In the lepton sector, there is one "symmetry-breaking" operator, or "spurion" in the language of [1] per vector space: $\mathbf{Y}_{\mathbf{e}} \mathbf{Y}_{\mathbf{e}}{ }^{\dagger}$ in $\ell_{L}$ space, $\mathbf{Y}_{\mathbf{e}}{ }^{\dagger} \mathbf{Y}_{\mathbf{e}}$ in $e_{R}$ space. These hermitian matrices can be diagonalised, and are uniquely identified by their eigenvalues in the eigenbasis. So we will sometimes say the operators can "choose a basis", and discuss interchangeably the matrix, the spurion and the basis of eigenvectors who are normalised to have length ${ }^{2}=$ the eigenvalue. In the presence of $\mathbf{Y}_{\mathbf{e}}$ (and the absence of other "basis choosing" operators in the lepton sector), there are three remaining global $U(1) \mathrm{s}$. The three conserved quantum numbers can be taken as the individual lepton flavours ${ }^{1}$. So in our restricted definition of the SM, neutrinos are massless and lepton flavours are conserved. We add neutrino masses at the end of the section.

In the quark sector, $\mathbf{Y}_{\mathbf{d}} \mathbf{Y}_{\mathbf{d}}^{\dagger}$ and $\mathbf{Y}_{\mathbf{d}}^{\dagger} \mathbf{Y}_{\mathbf{d}}$ choose respectively a basis in the $q_{L}$ and the $d_{R}$ flavour spaces. Similarly, $\mathbf{Y}_{\mathbf{u}} \mathbf{Y}_{\mathbf{u}}{ }^{\dagger}$ and $\mathbf{Y}_{\mathbf{u}}{ }^{\dagger} \mathbf{Y}_{\mathbf{u}}$ choose respectively a basis in the $q_{L}$ and the $u_{R}$ flavour spaces. So there are two operators in $q_{L}$ space, $\mathbf{Y}_{\mathbf{d}} \mathbf{Y}_{\mathbf{d}}{ }^{\dagger}$ and $\mathbf{Y}_{\mathbf{u}} \mathbf{Y}_{\mathbf{u}}{ }^{\dagger}$, who are not simultaneously diagonalisable. Flavour is therefore not conserved, and the misalignment between the two eigenbases is parametrised by the CKM matrix.

The mixing angles and phase of the quark sector are over-determined in many flavour-changing, flavour-conserving and CP violating processes of the quark sector. For instance, the CKM angles can be obtained in tree level processes, and used to predict rates that are mediated by loops in the Standard Model. To date, the experimentally measured rates agree with these predictions, implying that the new physics contribution in loops should be smaller than the SM. For new particles with generic flavour-changing couplings, this is a strong constraint, placing the mass above 10-100 $\mathrm{TeV}$ [10.

Minimal Flavour Violation was introduced to allow New Physics to have TeV-scale masses, and be consistent with precision flavoured data from the quark sector. It is a restriction on the flavour structure of new interactions. The only operators allowed in the "flavour-spaces" are those of the SM (and the identity matrix). So flavour-change and CP violation in the quarks are proportional to the CKM matrix and quark Yukawa eigenvalues, eg to $\mathbf{Y}_{\mathbf{d}} \mathbf{Y}_{\mathbf{d}}{ }^{\dagger}$ in the mass basis of up-type doublet quarks. MFV is therefore a predictive framework, and encompasses many of the models that fit the data.

Flavour-changing processes are also observed in the lepton sector, in neutrino oscillations. The weakly intereacting neutrinos are observed to have small mass differences, and large mixing angles with respect to the charged leptons. That is, in the lepton doublet space, there are two operators that break the flavour $U_{\ell}(3)$ symmetry. These are the charged lepton and neutrino mass matrices, which "choose bases" related by the MNS matrix $U$. In the charged lepton mass eigenstate basis (referred to as the "flavour" basis), the light neutrino mass matrix satisfies

$$
\left[m_{\nu}\right]\left[m_{\nu}\right]^{\dagger}=U^{*} D_{m_{\nu}}^{2} U^{T}
$$

where $D_{m_{\nu}}^{2}=\operatorname{diag}\left\{m_{1}^{2}, m_{2}^{2}, m_{3}^{2}\right\}$. To date, only flavour changing charged current processes (mediated by $W$ exchange) are observed in the lepton sector, and MFV is not "required" for the leptons. Four elements of the MNS matrix are measured - the remainder being obtained from unitarity [1] - and CP violation is not observed. This means new leptonic physics is not stringently constrained to agree with SM predictions for CP violation, as is the case in the quark sector. Rates for unobserved FCNC lepton processes (e.g. $\mu \rightarrow e \gamma$ ) can be calculated using the MNS matrix and neutrino masses, and are well below the current experimental sensitivity. So new leptonic physics is only constrained to be less than the experimental rates, and not, as in the quark sector, to be smaller than the prediction one obtains using observed masses.

The neutrino masses can be lepton number conserving ("Dirac") or not ("Majorana"). In the Dirac case one could define MFV in the lepton sector as an exact copy of the quarks, so in this paper, we consider Majorana neutrino

\footnotetext{
${ }^{1}$ The three $U(1)$ s can also be taken to correspond to the three diagonal generators of $U(3)=\left\{I, \lambda_{3}, \lambda_{8}\right\}$, acting simultaneously on the $\ell_{L}$ and $e_{R}$ flavour spaces. In this case one conserves the total lepton number $L_{e}+L_{\mu}+L_{\tau}$, and the flavoured asymmetries $L_{e}-L_{\mu}$ and $L_{e}+L_{\mu}-2 L_{\tau}$
} 
masses, which arise from a dimension five operator

$$
\left(\ell_{j} H_{u}\right) \mathbf{K}^{j k}\left(\ell_{k} H_{u}\right)
$$

Two classes of new physics generating this operator can be distinguished. One possibility is that it is generated by new flavoured particles, in a new flavour space. These new particles should be heavy or weakly coupled, since they have not been observed. The canonical example is the seesaw, where one adds, e.g., 3 generations of $\nu_{R}$, and the flavour symmetry group of the kinetic terms is enlarged to $U(3)^{6}$. The second possibility is that the all flavoured particles live in the 5 flavour spaces of the SM, and some new lepton number- or flavour-changing interactions are included. This is the case for neutrino masses generated in the R-parity violating MSSM.

\section{Minimal Flavour Violation for Leptons?}

We assume that there are new flavoured particles at the TeV scale, and hope that this is verified soon at the LHC. A definition of Minimal Flavour Violation in the lepton sector 3 could then be interesting for various reasons. Firstly, MFV in the quark sector is well motivated by the experimental observations. So one could conclude it reflects some principle or symmetry of the underlying theory, and should apply in the lepton sector as well. Secondly, in the lepton sector, we know there must be Beyond the Standard Model physics at some scale, because we observe neutrino masses. We can hope to use MFV as a tool in distinguishing among the multitude of candidate models for new physics in the lepton sector ${ }^{2}$. Minimal Flavour Violation for leptons should therefore be applicable to most models, and be predictive, so we can test the hypothesis and/or differentiate models.

A predictive definition of MFV for the lepton sector has recently been introduced in [3], and further studied in 12. It supposes that the three light neutrinos are Majorana, with the required lepton number violation occuring at some high scale $\Lambda_{L N}$. Two classes of models are considered: those whose particles transform according to the $U^{5}(3)$ flavour transformations of the SM, and a second scenarino with three (heavy) right-handed neutrinos.

In this work, we also take the light neutrino masses to be Majorana. If they were Dirac, MFV could be defined for leptons by copying the quark definition. Models that generate Majorana neutrino masses can be divided into two cases [3]:

- case A: models whose particles transform according to the $U^{5}(3)$ flavour transformations of the SM, and

- case B: models with a flavour transformation group that is larger than that of the SM (e.g. the seesaw, where the kinetic terms of the three $\nu_{R}$ have a $U(3)$ symmetry).

\subsection{Larger flavour transformation group}

Suppose there are a several generations of a new particle, e.g. three right-handed neutrinos. The kinetic terms therefore have an enlarged flavour symmetry group, which is $U^{6}(3)$ when $3 \nu_{R}$ are added to the SM. The renormalisable Lagrangian for the SM + the new particle will contain the SM Yukawas, and some number of additional spurions corresponding to the interactions of the new particle. In the case of the seesaw, the Lagrangian is

$$
\mathcal{L}_{S M}+\bar{\ell}_{j} \mathbf{Y}_{\nu}{ }^{j K} H_{u} N_{K}+\frac{1}{2} \overline{N_{J}^{c}} \mathbf{M}_{J J} N_{J}+\text { h.c. }
$$

and there are potentially two "basis-choosing" interactions, or spurions, in $\nu_{R}$ space: $\mathbf{Y}_{\nu}^{\dagger} \mathbf{Y}_{\nu}$ and $\mathbf{M}$. There are a variety of potential definitions of MFV, which we illustrate with the seesaw example.

1. one could impose that the new physics may not introduce new spurions in the Standard Model flavour spaces. In the case of the seesaw, this means that $\mathbf{Y}_{\nu} \mathbf{Y}_{\nu}^{\dagger}$ should be diagonal in the lepton doublet flavour basis (charged lepton mass eigenstate basis). No restrictions are imposed on the number of bases chosen in the flavour space of the new particles. In the seesaw case, $\mathbf{Y}_{\nu}^{\dagger} \mathbf{Y}_{\nu}$ and $\mathbf{M}$ could have different eigenbases, and must do so to reproduce the correct neutrino mixing angles. This definition of MFV for leptons is predictive but unattractive, because it implies that lepton flavour violation amoung charged leptons is suppressed by neutrino masses.

\footnotetext{
${ }^{2}$ Taking a principle of MFV to apply to the neutrino mass generation mechanism is a more ambitious implementation of MFV than in the quark sector. For quarks, one hopes for new TeV-scale particles (for instance to address the hierarchy problem), in which case MFV is almost required to describe the Lagrangian up to scales $\sim 100 \mathrm{TeV}$. In the lepton sector, we know there is New Physics, and it should have some connection to flavour because it generates neutrino masses. However, this new physics could be at a high scale $\left(\sim 10^{10}-10^{16}\right.$ $\mathrm{GeV}$ in the seesaw?), so in assuming that MFV applies to the interactions that generate the neutrino masses, we may be applying it across many more orders of magnitude than in the quark sector.
} 
2. CGIW [3] define MFV for the seesaw by allowing the renormalisable interactions of eqn (5) to choose a second basis in $\ell$ space, but impose restrictions on the spurions in $\nu_{R}$ space. They study the case where the $\nu_{R}$ are degenerate of mass $M$, and $\mathrm{CP}$ is a symmetry of the right-handed neutrino sector [13]. (So there is only one eigenbasis in $\nu_{R}$ space.)

In this case $\mathbf{K}=\mathbf{Y}_{\nu} \mathbf{M}^{-\mathbf{1}} \mathbf{Y}_{\nu}^{\mathbf{T}}=\mathbf{Y}_{\nu} \mathbf{Y}_{\nu}^{T} / M$. The two "basis-choosing" coupling matrices in $\ell$ space, that are relevant for lepton number conserving flavour violation, are

$$
\mathbf{Y}_{\mathbf{e}} \mathbf{Y}_{\mathbf{e}}^{\dagger}, \quad \mathbf{Y}_{\nu} \mathbf{Y}_{\nu}^{\dagger}=\frac{M}{v^{2}} \mathbf{U}^{*} \mathbf{D}_{m_{\nu}} \mathbf{U}^{T}
$$

If there is no CP violation in the lepton sector (the case studied by CGIW), then $\mathbf{Y}_{\nu} \mathbf{Y}_{\nu}^{\dagger}=M \mathbf{K}$. In either case, lepton flavour violation is controlled by parameters from the light neutrino sector. The predictions of this scenario should be similar to the SUSY seesaw with degenerate $\nu_{R}$ [14.

3. The more generic (and less predictive) definition of MFV for the seesaw would be to allow all renormalisable interactions to be independent spurions, as one allows for SM constituents (equivalently, one could allow up to two spurions per vector space). In the case of the $\nu_{R}$, with the seesaw Lagrangian of eqn (5D), the Majorana mass matrix $\mathbf{M}$ and the Yukawa coupling $\mathbf{Y}_{\nu}^{\dagger} \mathbf{Y}_{\nu}$ are independent spurions in the $\nu_{R}$ flavour space. Similarly to $\mathbf{Y}_{\nu} \mathbf{Y}_{\nu}^{\dagger}$ and $\mathbf{Y}_{\mathbf{e}} \mathbf{Y}_{\mathbf{e}}{ }^{\dagger}$ in $\ell$ space, they have unrelated eigenbases. This is the "usual" type-1 seesaw, whose supersymmetric flavour-changing predictions have been extensively studied in the literature [15]. It is well known that in the SUSY seesaw, the rates for flavour-changing processes among the charged leptons are not related to the neutrino masses or the MNS matrix [16].

\subsection{Standard Model flavour transformations}

Consider now neutrino mass models whose particles transform according to the $U^{5}(3)$ flavour transformations of the SM. In the quark sector, MFV restricts the bases chosen by flavour-dependent new interactions to be those of the SM Yukawas. That is, there are two allowed bases (spurions) in $q_{L}$ space, and one in $u_{R}$ and $d_{R}$ spaces respectively.

1. CGIW define MFV for leptons, in this case, to allow two spurions ("basis-choosing" operators) in the doublet lepton $(\ell)$ space, which are $\mathbf{K}$ and $\mathbf{Y}_{\mathbf{e}} \mathbf{Y}_{\mathbf{e}}{ }^{\dagger}$. The $\mathbf{K}$ is the dimensionful coefficient of a lepton number violating operator, so lepton flavour changing processes, that conserve lepton number, are controlled by the dimensionless $\Lambda_{L N}^{2} \mathbf{K K}^{\dagger}$. Rates for lepton flavour violating processes $(e . g . \mu \rightarrow e \gamma)$ are proportional to the unknown $\Lambda_{L N}^{2}$, but ratios of LFV processes are predicted to be controlled by $\mathbf{K K}^{\dagger}$. This describes for instance the SUSY triplet model [9, 3, 12].

2. Alternatively one could suppose that MFV is a restriction on renormalisable couplings. This is reasonable firstly because MFV is a recipe for extrapolating in scale. We know how renormalisable couplings evolve, whereas we cannot guess, in a bottom up approach, when a non-renormalisable interaction becomes renormalisable. Secondly, one could expect that flavour is introduced into the theory at some high scale, $\left(M_{G U T}\right.$ ?), and comes to us via renormalisable couplings.

- One could hope to define MFV, by analogy with the quark sector, as restricting all new interactions to be aligned with the SM Yukawas. But then it is difficult to obtain the large mixing angles of the MNS matrix. A model attempting to satisfy this ideal can be found in Appendix A. This version of MFV would predict that lepton flavour changing amplitudes must contain the neutrino mass to some power, or lepton number violation. Notice that this differs from the CGIW prediction; in the present case, lepton flavour violation is suppressed by the small neutrino mass scale.

- A more realistic definition of MFV, that includes some models, would allow (at least) one other basis in $\ell$ space. New renormalisable interactions can choose one, and only one, new basis for $\ell$ space, and no new bases for $\left\{e_{R}, u_{R}, d_{R}, q_{L}\right\}$ spaces. That is, we take MFV as a statement about renormalisable interactions, that allows two bases in the $q_{L}$ and $\ell$ spaces, one in the $u_{R}, d_{R}$ and $e_{R}$ spaces. The question then arises: is lepton flavour violation among charged leptons controlled by the light neutrino mass matrix? If yes, then this definition of MFVL is equivalent to that of CGIW. If the lepton flavour violating rates are independent, one could hope they give information about the neutrino mass generation mechanism. 
Some renormalisable, lepton number violating interactions involving $\ell$, that can be used to construct the neutrino mass matrix, are

$$
\begin{aligned}
\frac{1}{2} M_{T} \vec{T} \cdot \vec{T}^{\dagger}+g_{\phi} M_{T} H_{u}^{c} \vec{\tau} H_{u}^{c} \cdot \vec{T}+g_{i j} \bar{\ell}_{i}^{c} \vec{\tau} \ell_{j} \cdot \vec{T} & \text { (triplet) } \\
\mu_{i} L_{i} H_{u}+\lambda_{j r s}^{\prime} L_{j} Q_{r} D_{s}^{c}+\frac{1}{2} \lambda_{i j}^{n} L_{i} L_{j} E_{n}^{c} & \text { (R parity Violating) }
\end{aligned}
$$

where $H_{u}$ is the Standard Model doublet Higgs, $T$ is an $\mathrm{SU}(2)$ triplet scalar, and the second line is in superfield notation, so are renormalisable interactions in supersymmetry. Under the $U(3)$ flavour transformations of $\ell$ space, $g$ transforms as a symmetric $\mathbf{6}, \lambda^{\prime}$ and $\mu$ as $\overline{\mathbf{3}}$, and the antisymmetric $\lambda$ as a $\mathbf{3}$.

In the triplet model of eqn (7), the exchange of $\vec{T}$ induces the neutrino mass operator (4). The light neutrino mass matrix is therefore $\left[m_{\nu}\right]_{\alpha \beta} \propto g_{\alpha \beta}$, and flavour violation among the charged leptons is controlled by the light neutrino mass matrix [9]. In this model, this definition of MFV based on renormalisable couplings, agrees with the definition of CGIW based on mass matrices.

It seems not possible to generate observed light neutrino masses with the $\lambda^{\prime}$ coupling, if we implement strictly this definition of MFV. The $\lambda^{\prime}$ must respect MFV in the quark sector:

$$
\sum_{\ell, d} \lambda_{\ell q d}^{\prime} \lambda_{\ell p d}^{\prime *} \propto\left[\mathbf{Y}_{\mathbf{d}} \mathbf{Y}_{\mathbf{d}}^{\dagger}\right]_{q p} \quad \sum_{\ell, q} \lambda_{\ell q d}^{\prime} \lambda_{\ell q f}^{\prime *} \propto\left[\mathbf{Y}_{\mathbf{d}}^{\dagger} \mathbf{Y}_{\mathbf{d}}\right]_{d f}
$$

so the eigenvalues of $\lambda^{\prime}$ are those of the $\mathbf{Y}_{\mathbf{d}}$. This hierarchy, when combined with quark masses to obtain $m_{\nu}$, gives too steep a neutrino mass hierarchy. In the following section, we construct a neutrino mass model that satisfies this definition of MFV, using the $\lambda$ interaction.

\section{The $\lambda$ model}

The aim of this section is to construct a neutrino mass model that has two features. It should be minimally flavour violating, in the sense that the new renormalisable interaction $\lambda$ only introduces one new basis, or spurion, which is in $\ell_{L}$ space. And the model should agrees with current bounds on lepton flavour violating processes $(\mu \rightarrow e \gamma$, etc), but the predictions for these processes should not be determined by the light neutrino mass matrix.

We take the light neutrino masses to be generated entirely by the RPV $\lambda$ coupling, so we neglect $\lambda^{\prime}$ and bilinear $\mathrm{RPV}$. In the charged lepton mass eigenstate basis, the light neutrino mass matrix can be written [17.

$$
\left[m_{\nu}\right]_{i j}=\sum_{m, n, p, q} \lambda_{i n}^{m} \lambda_{p j}^{q} m_{e_{n}} \delta_{q}^{n} \tilde{A}^{m p} I\left(m_{\tilde{E}_{m}}, m_{\tilde{L}_{m}}\right)+(i \leftrightarrow j)
$$

where the $A$-term $\tilde{\mathbf{A}}^{m p}=-\left(\left(\mathbf{Y}_{\mathbf{e}} \mathbf{A}\right)^{m p} \frac{v_{d}}{\sqrt{2}}+\mu \frac{v_{u}}{\sqrt{2}} \mathbf{Y}_{\mathbf{e}}{ }^{m p}\right)$ is taken flavour diagonal and included in the mass insertion approximation, the mass matrices for the sleptons $\tilde{E}_{m}$ and $\tilde{L}_{m}$ are taken diagonal in the flavour basis (which is consistent with MFV), and

$$
I\left(m_{1}, m_{2}\right)=-\frac{1}{16 \pi^{2}} \frac{m_{1}^{2}}{m_{1}^{2}-m_{2}^{2}} \ln \frac{m_{1}^{2}}{m_{2}^{2}} .
$$

The $\lambda_{i j}^{n}$ is an antisymmetric matrix on its doublet indices $i, j$, so corresponds to a plane in $\ell_{L}$ space. It is convenient to rewrite it as a single index object in $\ell_{L}$ space (the vector orthogonal to the plane), using the antisymmetric $\epsilon$ tensor

$$
\tilde{\lambda}_{n k}=\frac{1}{2} \epsilon_{i j k} \lambda_{i j}^{n} .
$$

The $\epsilon_{i j k}$ is $\mathrm{SU}(3)$ invariant, but not $U(3)$ invariant, so this renaming has some peculiar consequences. Consider the case where $\lambda_{i j}^{n} \propto \epsilon_{i j n}$, so $\tilde{\lambda}$ is "flavour diagonal". However, since it transforms under $S U_{\ell}(3) \times S U_{e}(3)$ as $\ell e_{R}$, it is not invariant, and the flavour differences $L_{e}-L_{\mu}$ and $L_{e}+L_{\mu}-2 L_{\tau}$ are conserved mod 2 in four fermion interactions.

The "MFV" constraint is that $\sum_{j} \tilde{\lambda}_{s j} \tilde{\lambda}_{t j}^{*}$ should be diagonal in the singlet charged lepton mass basis, with eigenvalues proportional to the charged lepton Yukawas ${ }^{3}$. We will permute the $\mu-\tau$ eigenvalues, that is $\sum_{i j} \lambda_{i j}^{e} \lambda_{i j}^{e *} \propto m_{e}^{2} / v^{2}$,

\footnotetext{
${ }^{3}$ Since the SM has only one eigenbasis in $e_{R}$ space, it is not required of new interactions that they have the same eigenvalues as $\mathbf{Y}_{\mathbf{e}}{ }^{\dagger} \mathbf{Y}_{\mathbf{e}}$, provided that they have the same eigenvectors.
} 

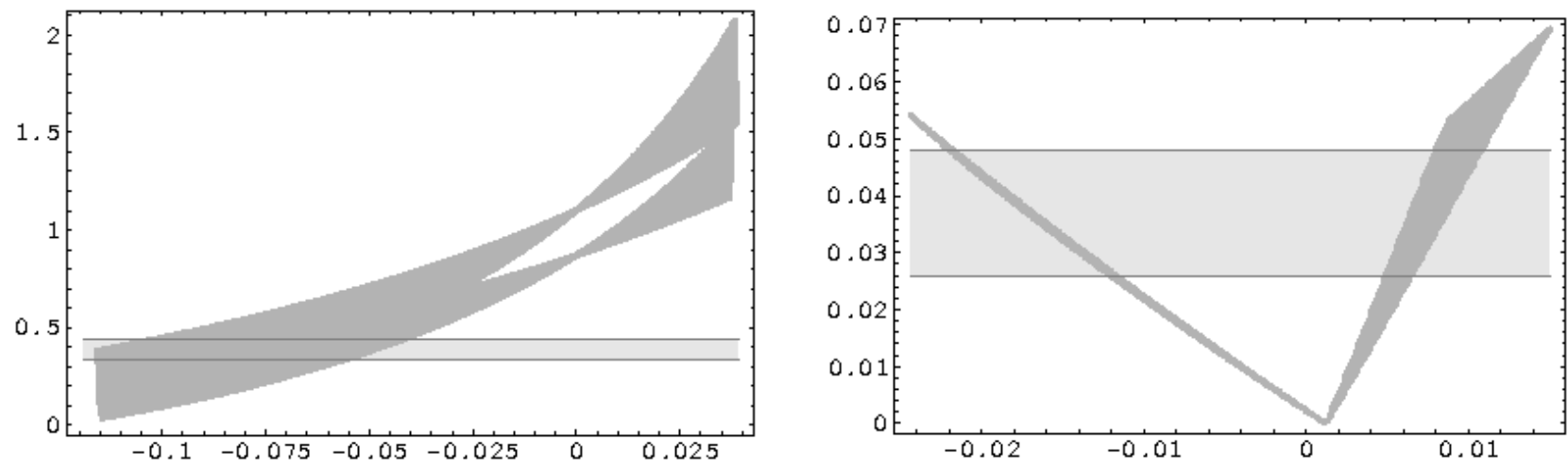

Figure 1: For slepton masses at $\sim 300 \mathrm{GeV}$ and $\tan \beta \sim 25$, on the left we plot our prediction for the "solar" mixing parameter $\tan ^{2} \theta_{12}$ as a function of the model parameter $\delta$ (see eqn (13)); where we have choosen an $\epsilon$ such that the other parameters $\left(\Delta m_{\text {sol }}^{2} / \Delta m_{a t m}^{2}, \tan ^{2} \theta_{23}, \sin ^{2} \theta_{13}\right)$ satisfy the experimental bounds. On the right we plot the ratio $\Delta m_{\text {sol }}^{2} / \Delta m_{\text {atm }}^{2}$ as a function of $\epsilon$, with $\delta$ consistent with the experimental bounds on the other physical parameters $\left(\tan ^{2} \theta_{12}, \tan ^{2} \theta_{23}, \sin ^{2} \theta_{13}\right)$. In both cases the horizontal light gray band represents the experimentally allowed parameter space [18].

but $\sum_{i j} \lambda_{i j}^{\mu} \lambda_{i j}^{\mu *} \propto m_{\tau}^{2} / v^{2}$, and $\sum_{i j} \lambda_{i j}^{\tau} \lambda_{i j}^{\tau *} \propto m_{\mu}^{2} / v^{2}$. On its doublet indices

$$
V_{\lambda}^{\dagger} \tilde{\lambda} \tilde{\lambda}^{\dagger} V_{\lambda}=\operatorname{diag}\left\{m_{e}^{2}, m_{\tau}^{2}, m_{\mu}^{2}\right\} / v^{2}
$$

where $V_{\lambda}$ is a unitary matrix transforming from the charged lepton basis to the eigenbasis of $\tilde{\lambda}$. The observed light neutrino parameters, with masses in the inverse hierarchy, can be obtained from

$$
V_{\lambda}^{\dagger}=\left[\begin{array}{ccc}
-c \epsilon & \frac{1+s \epsilon}{\sqrt{2}} & -\frac{1-s \epsilon}{\sqrt{2}} \\
s & \frac{c}{\sqrt{2}} & \frac{c}{\sqrt{2}} \\
c & -\frac{s-\epsilon}{\sqrt{2}} & -\frac{s+\epsilon}{\sqrt{2}}
\end{array}\right]
$$

where $c=\cos (\pi / 4+\delta)$. This corresponds to

$$
\begin{aligned}
\lambda_{\alpha \beta}^{e} \propto \frac{m_{e}}{v} \sim 0 & \\
\lambda_{\mu \tau}^{\mu}=s \frac{m_{\tau}}{v} & \lambda_{e \tau}^{\mu}=\frac{c}{\sqrt{2}} \frac{m_{\tau}}{v} \quad \lambda_{e \mu}^{\mu}=\frac{c}{\sqrt{2}} \frac{m_{\tau}}{v} \\
\lambda_{\mu \tau}^{\tau}=c \frac{m_{\mu}}{v} & \lambda_{e \tau}^{\tau}=-\frac{s-\epsilon}{\sqrt{2}} \frac{m_{\mu}}{v} \quad \lambda_{e \mu}^{\tau}=-\frac{s+\epsilon}{\sqrt{2}} \frac{m_{\mu}}{v}
\end{aligned}
$$

For $\theta=\pi / 4, \epsilon=0$ and degenerate sleptons, eqn (9) gives exactly degenerate neutrinos $\nu_{e}$ and $\nu_{\mu-\tau}$, whose mass varies inversely with the slepton mass. The observed neutrino mass differences and mixing angles, in the inverse hierarchy, can be obtained by including small perturbations. e difference between the square of the slepton masses $\tilde{m}_{\mu}^{2}-\tilde{m}_{\tau}^{2}$ and the small mixing angle $\epsilon$ contribute to splitting the $\nu_{1}$ and $\nu_{2}$ masses, while the parameter $\delta$ of the $V_{\lambda}$ matrix seems to control the solar mixing angle.

In Figure (11) we show the behaviour of two physical parameters, $\tan ^{2} \theta_{12}$ and the ratio $\Delta m_{\text {sol }}^{2} / \Delta m_{\text {atm }}^{2}$, when the parameters $\delta$ and $\epsilon$ vary. For both the plots, we have considered only those points in agreement with the experimental bounds on the remaining set of neutrino parameters. From the intersection between the dark and the light region we can deduce the range of availability for $\epsilon$ and $\delta$. (The slepton mass difference in these plots is fixed at a value that could be generated by renormalisation group running.)

This $\lambda$ model, then, satisfies our requests. The neutrino masses are generated by a renormalisable operator $\tilde{\lambda}$, whose eigenbasis is related with the charged lepton mass eigenbasis by a matrix $V_{\lambda}^{\dagger}$ different from the MNS mixing matrix. The matrix $V_{\lambda}^{\dagger}$, then, has become the operator that guides flavour violating processes, whose amplitudes are now determined by the $\lambda$ couplings. In particular, we can see in (14) that the order of magnitude of each $\lambda$ coupling is determined by its upper index, which is related with the flavour of the right-handed particle involved in the vertex. 


\begin{tabular}{|l|c|c|}
\hline & Expected value & Experimental bound \\
\hline \hline$B R\left(\mu^{-} \rightarrow e^{-} e^{+} e^{-}\right)$ & $\sim 10^{-17\left(\frac{100 \mathrm{GeV}}{m_{\tilde{\nu}}}\right)^{4}}$ & $<1.010^{-12}$ \\
$B R\left(\mu^{-} \rightarrow e^{-} \gamma\right)$ & $\sim 10^{-12\left(\frac{100 \mathrm{GeV}}{m_{\tilde{\nu}}}\right)^{4}}$ & $<1.210^{-11}$ \\
$B R\left(\tau^{-} \rightarrow e^{-} e^{+} e^{-}\right)$ & $\sim 10^{-19}\left(\frac{100 \mathrm{GeV}}{m_{\tilde{\nu}}}\right)^{4}$ & $<2.910^{-6}$ \\
$B R\left(\tau^{-} \rightarrow e^{-} \mu^{+} \mu^{-}\right)$ & $\sim 10^{-10\left(\frac{100 \mathrm{GeV}}{m_{\tilde{\nu}}}\right)^{4}}$ & $<1.810^{-6}$ \\
$B R\left(\tau^{-} \rightarrow e^{+} \mu^{-} \mu^{-}\right)$ & $\sim 10^{-12\left(\frac{100 \mathrm{GeV}}{m_{\tilde{\nu}}}\right)^{4}}$ & $<1.510^{-6}$ \\
$B R\left(\tau^{-} \rightarrow \mu^{-} e^{+} e^{-}\right)$ & $\sim 10^{-12\left(\frac{100 \mathrm{GeV}}{m_{\tilde{\nu}}}\right)^{4}}$ & $<1.710^{-6}$ \\
$B R\left(\tau^{-} \rightarrow \mu^{+} e^{-} e^{-}\right)$ & 0 & $<1.510^{-6}$ \\
$B R\left(\tau^{-} \rightarrow \mu^{-} \mu^{+} \mu^{-}\right)$ & $\sim 10^{-11\left(\frac{100 \mathrm{GeV}}{m_{\tilde{\nu}}}\right)^{4}}$ & $<1.910^{-6}$ \\
\hline
\end{tabular}

Table 1: Table of the branching ratios for flavour violating processes. In the second column appear the branching ratios predicted in the $\lambda$ model, while in the third column are indicate the experimental bounds at $90 \%$ of confidence level [19.

As we can see in Table 1 the experimental bounds on FV decays are satisfied in this $\lambda$ model, in agreement with our definition of MFV.

The strongest experimental constraints on FV processes are given for the muon decay into three electrons and the $\mu \rightarrow e \gamma$ decay [19, 20, 21]. The flavour violating decays with charged leptons in the initial and final states, like $\mu^{-} \rightarrow e^{-} e^{+} e^{-}$, appear at the tree level and are mediated by the exchange of a left-handed sneutrino $\tilde{\nu}_{i}$. So, it can be easily understood why the decay rates of muon, but also tau, into three electrons are so low. In addition to the suppression due to the sneutrino mass, in each diagram appears a vertex $\tilde{\nu} e e$, whose amplitude is determined by a coupling of the form $\lambda_{\alpha \beta}^{e}$ which is proportional to the small electron mass, since right-handed sneutrinos are not present in the model.

The $\mu \rightarrow e \gamma$ decay [20], instead, appears at a loop level, mediated by a charged lepton and slepton. In this case the main contribution comes from the diagram with vertices proportional to $\lambda_{\mu \tau}^{\mu} \lambda_{e \tau}^{\mu} \propto m_{\tau}^{2} / v^{2}$, whose large contribution is somewhat compensated by the loop suppression. We estimate the decay branching ratio in our model to be $\sim 6 \times 10^{-13}$, and we can notice that, although this value respects the present experimental constraint, it could be accessible in the next experiments.

\section{Summary}

In the lepton sector, new beyond-the-Standard-Model (BSM) interactions are required to generate neutrino masses. If these masses are Majorana, they arise from a dimension five operator whose flavour structure (eigenvalues, eigenvectors in lepton doublet space) may not be the same as the (renormalisable) BSM interactions. In this context, it is not obvious to define Minimal Flavour Violation for leptons. One can take the "minimal" scenario to be that flavourchange in the lepton sector is controlled by the neutrino mass matrix, which is in part known. This predictive approach was taken in 3 .

However, there are neutrino mass generation mechanisms that do not make this prediction, since flavour change may be proportional to a different combination of renormalisable couplings than enters $m_{\nu}$. In this paper, we explore various possible definitions of "minimally flavour violating", based on the renormalisable interactions in the Lagrangian. We suppose that Minimal Flavour Violation is a restriction on the number of inequivalent eigenbases that renormalisable flavour-dependent interactions can choose. The most minimal possibility would be to restrict the new interactions to align themselves with the charged lepton Yukawa, but then it is difficult to obtain MNS mixing angles (without enlarging the flavour transformation group, for instance by adding right-handed neutrinos). The leptonic masses and 
mixing angles can be obtained in more models (e.g. triplet, R-parity violation) by allowing two eigenbases in doublet lepton space. The second basis may be other than the neutrino mass basis; we construct a model where flavour violation among charged leptons is not predictable from the light neutrino mass matrix.

Data in the quark sector suggest that new particles and interactions at the TeV-scale should satisfy Minimal Flavour Violation. Data in the lepton sector do not require a minimal flavour violation principle, but one could imagine it is there, by analogy with the quarks. Unfortunately, there are many possible definitions, which seem either predictive, or able to include many models. A compelling definition of MFV, giving different predictions for different neutrino mass generation mechanisms, could be useful in attempting a bottom-up reconstruction of the neutrino mass mechanism 22] from lepton flavour violating rates.

\section{Appendix A}

The aim of this appendix is to obtain an acceptable neutrino mass matrix, using new interactions that are diagonal in the charged lepton mass basis. We consider an RPV model, with lepton number violating terms in the superpotential

$$
\frac{1}{2} \lambda_{i j}^{k} L_{i} L_{j} E_{k}^{c}+\mu_{i} H_{u} L_{i}
$$

an $\Psi$ soft term $B_{i} H_{u} L_{i}$, and we estimate the light neutrino mass matrix from the formulae in 23. It seems possible to obtain degenerate light neutrinos $\left(m_{\nu} \sim 0.2 \mathrm{eV}\right)$, and an MNS matrix in agreement with observations ${ }^{4}$.

This peculiar result arises by taking

$$
\lambda_{i j}^{k}=\lambda \epsilon_{i j k} \quad, \quad \mu_{\mu}=\delta_{\mu} \mu_{0} \quad, \quad B_{\tau}=\delta_{B} B_{0}
$$

and all other $R_{p}$ couplings to be zero. The usual $\mu_{0}$ and $B_{0}$ terms are $\mu_{0} H_{u} H_{d}$ in the superpotential and $B_{0} H_{u} H_{d}$ among the soft breaking terms, and we will later solve for the desired values of $\delta_{\mu}, \delta_{B}$. We claim that $\lambda \propto \epsilon_{i j k}$ is "flavour-diagonal", insofar as it is an SU(3) invariant (see discussion after eqn (11)). We can obtain off-diagonal contributions to the neutrino mass matrix, by combining it with the "bilinear" $R_{p}$ interactions $B_{\tau}$ and $\mu_{\mu}$.

The leading contributions to the neutrino mass matrix, in the charged lepton mass basis, can be estimated as [23.

$$
\left[m_{\nu}\right] \simeq \frac{1}{8 \pi^{2} m_{S U S Y}}\left[\begin{array}{ccc}
\lambda^{2} m_{\mu} m_{\tau} & \lambda \delta_{B} m_{\tau}\left(h_{\mu} m_{\mu}-h_{e} m_{e}\right) & \lambda \delta_{\mu} m_{\mu}\left(h_{e} m_{e}-h_{\tau} m_{\tau}\right) \\
\lambda \delta_{B} m_{\tau}\left(h_{\mu} m_{\mu}-h_{e} m_{e}\right) & 8 \pi^{2}\left|\delta_{\mu}\right|^{2} m_{S U S Y}^{2} & g^{2} \delta_{\mu} \delta_{B} m_{S U S Y}^{2} / 8 \\
\lambda \delta_{\mu} m_{\mu}\left(h_{e} m_{e}-h_{\tau} m_{\tau}\right) & g^{2} \delta_{\mu} \delta_{B} m_{S U S Y}^{2} / 8 & g^{2} \delta_{B}^{2} m_{S U S Y}^{2} / 8
\end{array}\right]
$$

where $m_{S U S Y} \sim 300 \mathrm{GeV}$ is of order the slepton and neutralino masses.

We can match this onto the neutrino mass matrix for degenerate light neutrinos, with $\theta_{13}=0$. Concentrating first on the $\mu \tau$ submatrix, we obtain

$$
\delta_{\mu} \simeq \sqrt{\frac{m_{1}}{m_{S U S Y}}} \quad \delta_{B} \simeq \frac{8 \pi}{g} \sqrt{\frac{m_{1}}{m_{S U S Y}}}
$$

and get the large atmospheric mixing by taking $m_{1}$, the lightest mass of the degenerate neutrinos, to be $\sqrt{4 \pi \Delta m_{a t m}^{2} / g^{2}}$ $\simeq .2 \mathrm{eV}$.

The first row has a desirable sign difference between the $e \mu$ and $e \tau$ entries, and can be adjusted to give the solar mass difference and mixing angle by taking $\lambda \sim .02$ and $\tan \beta \gtrsim 10(\tan \beta$ enters via the charged lepton Yukawas).

\section{Acknowledgements}

We thank Aldo Deandrea, Gino Isidori, and Alessandro Strumia for discussions.

\section{References}

[1] G. D'Ambrosio, G. F. Giudice, G. Isidori and A. Strumia, Nucl. Phys. B 645 (2002) 155 arXiv:hep-ph/0207036.

\footnotetext{
${ }^{4}$ we did not scan parameter space, more realistic masses could be possible. Our example requires delicate fine-tuning.
} 
[2] A. J. Buras, P. Gambino, M. Gorbahn, S. Jager and L. Silvestrini, Phys. Lett. B 500 (2001) 161 arXiv:hep-ph/0007085. C. Bobeth, M. Bona, A. J. Buras, T. Ewerth, M. Pierini, L. Silvestrini and A. Weiler, Nucl. Phys. B 726 (2005) 252 arXiv:hep-ph/0505110.

[3] V. Cirigliano, B. Grinstein, G. Isidori and M. B. Wise, Nucl. Phys. B 728 (2005) 121 arXiv:hep-ph/0507001.

[4] K. S. Babu and C. N. Leung, Nucl. Phys. B 619 (2001) 667 arXiv:hep-ph/0106054. M. C. Gonzalez-Garcia and Y. Nir, Rev. Mod. Phys. 75 (2003) 345 arXiv:hep-ph/0202058.

[5] P. Minkowski, Phys. Lett. B 67 (1977) 421; M. Gell-Mann, P. Ramond and R. Slansky, Proceedings of the Supergravity Stony Brook Workshop, New York 1979, eds. P. Van Nieuwenhuizen and D. Freedman; T. Yanagida, Proceedinds of the Workshop on Unified Theories and Baryon Number in the Universe, Tsukuba, Japan 1979, ed.s A. Sawada and A. Sugamoto; R. N. Mohapatra, G. Senjanovic, Phys.Rev.Lett. 44 (1980)912.

[6] H.P. Nilles and N. Polonsky, Nucl. Phys. B499 (1997) 33. T. Banks, Y. Grossman, E. Nardi and Y. Nir, Phys. Rev. D52 (1995) 5319. L. Hall and M. Suzuki. Nucl. Phys., B231:419, 1984. Y. Grossman and H. Haber, Phys. Rev. Lett. 78 (1997) 3438; Phys.Rev. D59 093008; hep-ph/9906310 R. Hempfling Nucl.Phys. B478 (1996) 3. Eung Jin Chun, Sin Kyu Kang, Phys.Rev. D61 (2000) 075012, M. Hirsch, M.A. Diaz, W. Porod, J.C. Romao, J.W.F. Valle, hep-ph/0004115

[7] A. Zee, Phys. Lett. B 93 (1980) 389 [Erratum-ibid. B 95 (1980) 461].

[8] G. B. Gelmini and M. Roncadelli, Phys. Lett. B 99 (1981) 411. J. Schechter and J. W. F. Valle, Phys. Rev. D 25 (1982) 774. A. Santamaria, Phys. Rev. D 39 (1989) 2715. K. Choi and A. Santamaria, Phys. Lett. B 267 (1991) 504. C. Wetterich, Nucl. Phys. B 187 (1981) 343.

[9] A. Rossi, Phys. Rev. D 66 (2002) 075003 arXiv:hep-ph/0207006.

[10] see e.g. A. J. Buras, arXiv:hep-ph/0505175

[11] S. Antusch, C. Biggio, E. Fernandez-Martinez, M. B. Gavela and J. Lopez-Pavon, arXiv:hep-ph/0607020

[12] V. Cirigliano and B. Grinstein, arXiv:hep-ph/0601111 V. Cirigliano, G. Isidori and V. Porretti, arXiv:hep-ph/0607068

[13] G. C. Branco, M. N. Rebelo and J. I. Silva-Marcos, Phys. Rev. Lett. 82 (1999) 683 arXiv:hep-ph/9810328.

[14] J. Hisano, T. Moroi, K. Tobe and M. Yamaguchi, Phys. Rev. D 53 (1996) 2442 arXiv:hep-ph/9510309.

[15] an incomplete selection of papers studying LFV in the seesaw (see also [14):

J. Hisano and D. Nomura, Phys. Rev. D 59 (1999) 116005 arXiv:hep-ph/9810479.

S. Lavignac, I. Masina and C. A. Savoy, Nucl. Phys. B 633 (2002) 139 arXiv:hep-ph/0202086.

J. A. Casas and A. Ibarra, Nucl. Phys. B 618 (2001) 171 arXiv:hep-ph/0103065.

[16] S. Davidson and A. Ibarra, JHEP 0109 (2001) 013 arXiv:hep-ph/0104076.

[17] S. Davidson and M. Losada, Phys. Rev. D 65 (2002) 075025 arXiv:hep-ph/0010325.

[18] B. Aharmim et al. [SNO Collaboration], Phys. Rev. C 72 (2005) 055502 arXiv:nucl-ex/0502021. T. Araki et al. [KamLAND Collaboration], Phys. Rev. Lett. 94 (2005) 081801 arXiv:hep-ex/0406035. J. Nelson, for the Minos Experiment, talk at Neutrino'06, Santa Fe. Y. Ashie et al. [Super-Kamiokande Collaboration], "A measurement of atmospheric neutrino oscillation parameters by Phys. Rev. D 71 (2005) 112005 arXiv:hep-ex/0501064.

[19] W.-M. Yao et al., J. Phys. G 33, 1 (2006)

[20] M. L. Brooks et al. [MEGA Collaboration], Phys. Rev. Lett. 83 (1999) 1521 arXiv:hep-ex/9905013.

[21] U. Bellgardt et al. [SINDRUM Collaboration], Nucl. Phys. B 299 (1988) 1.

[22] M. Pospelov, A. Ritz and Y. Santoso, Phys. Rev. Lett. 96 (2006) 091801 arXiv:hep-ph/0510254. E. J. Chun, AIP Conf. Proc. 805 (2006) 145 arXiv:hep-ph/0510318.

[23] S. Davidson and M. Losada, JHEP 0005, 021 (2000) arXiv:hep-ph/0005080. 